1Fundação Oswaldo Cruz (Fiocruz), Instituto Nacional de Controle de Qualidade em Saúde (INCQS), Departamento de Imunologia - Rio de Janeiro (RJ), Brasil. gabriele.souza@incqs. fiocruz.br

2 Fundação Oswaldo Cruz (Fiocruz), Escola Nacional de Saúde Pública Sergio Arouca (Ensp), Departamento de Direitos Humanos, Saúde e Diversidade Cultural (DIHS) - Rio de Janeiro (RJ), Brasil.

aldopachecoferreira@gmail. com

${ }^{3}$ Fundação Oswaldo Cruz (Fiocruz), Escola Nacional de Saúde Pública Sergio Arouca (Ensp), Centro de Estudos da Saúde do Trabalhador e Ecologia Humana (Cesteh) - Rio de Janeiro (RJ), Brasil. mfr.moreira55@gmail.com

4 Fundação Oswaldo Cruz (Fiocruz), Instituto Oswaldo Cruz (IOC) - Rio de Janeiro (RJ), Brasil. luportela@yahoo.com

\section{Fatores de riscos ocupacionais e implicações à saúde do trabalhador em biotérios}

\author{
Occupational risk factors and implications for vivarium workers' \\ health
}

Gabriele Fatima de Souza', Aldo Pacheco Ferreira², Maria de Fátima Ramos Moreira ${ }^{\mathbf{3}}$, Luciana

Fernandes Portela 4

RESUMO Estudo transversal que avaliou as implicações à saúde dos trabalhadores de biotérios, bem como fatores de risco inerentes, em uma Instituição Federal de Ciências e Tecnologia. Os dados foram coletados por meio de um questionário semiestruturado e observação participante, em 2014. Foi evidenciado que os trabalhadores dos biotérios pesquisados em geral têm suas funções relacionadas com os fatores de risco físico, de acidente, ergonômico, biológico e organizacional/psicossocial. A pesquisa refletiu o quadro atual de deterioração das relações trabalho-saúde, sinalizando a necessidade de atualização constante no monitoramento na atenção à saúde desses trabalhadores.

PALAVRAS-CHAVE Fatores de risco. Saúde do trabalhador. Técnicos em manejo de animais. Ambiente de trabalho.

\begin{abstract}
Cross-sectional study that assessed the implications for vivarium workers' health and inherent risk factors in a Federal Institution of Science and Technology. Data were collected through a semi-structured questionnaire and participant observation in 2014. It was demonstrated that the vivarium workers' surveyed in general have their functions related to physical, accidents, ergonomic, biological, chemical and organizational/psychosocial risk factors. The survey reflected the current situation of deterioration of the work-health relation at vivariums, signaling the need to update and constant monitoring in the health care of these workers.
\end{abstract}

KEYWORDS Risk factors. Occupational health. Animal technicians. Working environment. 


\section{Introdução}

Embora nem todas as pesquisas envolvam o uso de animais, essa prática ainda é essencial em muitas áreas. Apesar dos avanços em relação aos métodos alternativos, nas condições atuais, os animais são insubstituíveis em muitas circunstâncias. Dessa forma, entende-se ser necessário o estabelecimento de uma cultura de cuidados, consciência e responsabilidade dirigidos à melhoria dos testes científicos, do bem-estar dos animais, bem como da proteção dos profissionais que desenvolvem as atividades no ambiente em que são produzidos e mantidos esses animais: o biotério (CARDOSO; SILVA, 2007).

Os profissionais de Biotério, ambiente de trabalho foco deste estudo, estão o tempo todo submetidos a uma série de riscos químicos, físicos, ergonômicos, organizacionais e de acidentes, acrescidos daqueles representados por agentes biológicos, uma vez que se expõem constantemente ao contato com sangue e outros fluidos orgânicos contaminados por uma variedade imensa de patógenos, sendo todos potenciais desencadeadores de doenças ocupacionais (MAJEROWICZ, 2008A; FELASA, 2010; NRC, 2010). Assim, esse ambiente pode, a qualquer momento, se transformar em um local hostil, por ter esse combinado de fatores de riscos que atuam direta ou indiretamente no processo do trabalho, interferindo na segurança, na saúde dos profissionais e no resultado do próprio trabalho (MEZADRI; TOMAZ; AMARAL, 2004; COSTA, 2008).

Por esse fato e, tendo em vista a relevância dessa temática no contexto atual, este artigo busca contribuir para o conhecimento no âmbito do trabalho em biotérios, auxiliando nos avanços da compreensão dos impactos do trabalho e nas políticas de intervenção direcionadas à saúde do trabalhador. Para tal, teve como objetivo analisar a associação entre a finalidade dos biotérios e os fatores de riscos ocupacionais, bem como as implicações à saúde do trabalhador nos biotérios de uma Instituição Federal de Ciências e
Tecnologia, tendo em vista a percepção dos profissionais de biotérios. Para isso, considerou-se fundamental a incorporação efetiva dos trabalhadores como atores desse processo, pois, como sujeito de sua vida e de sua saúde, é capaz de incorporar suas vivências e conhecimentos adquiridos ao longo de suas trajetórias, já que realizam seu trabalho cotidianamente e sofrem com seus efeitos (PORTO, 2000; NOUROUDINE, 2004).

Neste estudo, a preocupação com a saúde fundamentou-se como resultado de diversas análises com as atividades de técnicos em manejo de animais, principalmente na construção do entendimento da relação do trabalho-saúde, que se configurou de diferentes formas de acordo com o contexto operacional de cada biotério pesquisado.

\section{Metodologia}

Trata-se de um estudo transversal, recorte de uma pesquisa de mestrado que ocorreu no período de março a outubro de 2014. Participaram do estudo 151 profissionais de ambos os sexos, que atuavam há pelo menos 6 meses nas áreas finalísticas dos biotérios de uma instituição federal de ciências e tecnologia. Cabe ressaltar que foram considerados profissionais das áreas finalísticas aqueles que trabalham diretamente com os animais na criação, manutenção e experimentação ou na lavagem de materiais e higienização de ambiente interno no biotério.

Para a coleta de dados, foi utilizado um questionário semiestruturado, multidimensional e autopreenchível, que continha informações sobre as características sociodemográficas e profissionais, identificação dos fatores de risco presentes no ambiente de trabalho e questões sobre problemas/ implicações à saúde. Esse instrumento foi formulado a partir da adaptação dos questionários desenvolvidos por Pere Boix e Laurent Vogel (2000) no 'Guia de avaliação de risco no local de trabalho’ e aprimorado após 
estudo piloto, envolvendo quatro técnicos em manejo de animais de unidades distintas. A pesquisa contemplou, em todas as suas etapas, as normas estabelecidas pela Portaria $n^{0}$ 466/2012 do Ministério da Saúde, sendo submetida e aprovada pelo Comitê de Ética da Ensp/Fiocruz (Escola Nacional de Saúde Pública Sergio Arouca/Fundação Oswaldo Cruz) sob o parecer consubstanciado 528.931 e CAAE 126441614.9.0000.5240.

A variável considerada como fator de exposição foi a 'finalidade do biotério'. Para formação dessa variável, os participantes foram agrupados em duas categorias definidas em função das atividades dos biotérios que trabalhavam, a saber: biotério de experimentação e biotério de criação/manutenção.
As variáveis de desfecho foram os 'fatores de risco' e as 'implicações à saúde'. A primeira trata-se de variáveis dicotômicas, cujas opções de respostas foram 'sim' ou 'não', sendo divididas com base na natureza de cada fator de risco (físico, químico, biológico, acidente, ergonômico e organizacional/psicossocial). Para sua categorização, utilizou-se como ponto de corte a mediana do total de itens relacionados a cada fator de risco. Como observado no quadro 1, considerou-se 'alto risco' a soma de valores de questões positivas com resultados acima da mediana, e 'baixo risco' a soma de valores de questões positivas com resultado abaixo ou igual à mediana; sendo questões positivas todas aquelas que tiveram a opção 'sim' com a resposta.

Quadro 1. Categorização das variáveis referentes aos fatores de risco a partir da mediana. Saúde do trabalhador em biotérios, 2014

\begin{tabular}{lllll}
\hline Fatores de risco & Baixo risco & Alto risco & Total de itens & Mediana \\
\hline Físico & 0 a 2 respostas positivas & 3 a 6 respostas positivas & 6 & 2 \\
Biológico & 0 a 2 respostas positivas & 3 respostas positivas & 3 & 2 \\
Químico & 0 a 2 respostas positivas & 3 a 5 respostas positivas & 4 & 2 \\
Acidente & 0 a 6 respostas positivas & 7 a 15 respostas positivas & 15 & 6 \\
Ergonômico & 0 a 5 respostas positivas & 6a 8 respostas positivas & 8 & 5 \\
Organizacional & 0 a 5 respostas positivas & 6a 13 respostas positivas & 13 & 5 \\
Psicossocial & & & \\
\hline
\end{tabular}

Fonte: Elaboração própria.

Já o desfecho 'Implicações à saúde’ trata-se de uma variável originalmente composta por uma lista de danos/problemas de saúde apresentados ou não pelos trabalhadores (resposta= sim ou não). Quando presentes, poderiam ser 'provocados', 'agravados' ou 'não possuir' relação com o trabalho (Provocados: o trabalhador não possuía o problema de saúde antes de trabalhar no biotério e adquiriu devido às atividades que realiza. Agravados: o trabalhador já tinha o problema de saúde antes de trabalhar no biotério e ficou pior devido as atividades que realiza. Não possui: o trabalhador tem ou teve o agravo e o mesmo não está relacionado com as atividades exercidas no biotério). Para este estudo, essas variáveis foram agrupadas em duas categorias definidas em função da presença e relação com o trabalho: sem a implicação (respondeu não) e provocada/agravada pelo trabalho (respondeu sim + provocado e/ou agravado). Nessa categorização, foram excluídos os profissionais que, apesar de relatarem alguma das implicações à saúde, consideraram não ter 
relação com o trabalho, pois a ideia dessa etapa é comparar quem não tem determinada implicação à saúde com quem tem e considerou ser provocado e/ou agravado pelo trabalho. Cabe enfatizar que as respostas foram referentes à percepção dos bioteristas que participaram da pesquisa.

Os dados foram analisados utilizando o programa SPSS versão 19. As associações entre as variáveis de exposição e desfecho foram testadas por meio de análises bivariadas utilizando o teste de Qui-quadrado de Pearson com níveis de significância de 5\% $(p=0,05)$. Para analisar os resultados das variáveis que não cumpriram os pré-requisitos para uso do Qui-quadrado de Pearson, foram adotados os valores do teste exato de Fisher.

\section{Resultados}

A amostra estudada foi composta por 151 profissionais das áreas finalísticas, que corresponde a $88,8 \%$ dos elegíveis. Destes, 59,6\% eram do sexo masculino; $34,3 \%$ tinham idade média de 38 anos DP \pm 10 anos, $51 \%$ eram casados ou viviam em união estável e 41,7\% tinham nível médio/técnico. Com relação ao perfil laboral, $51 \%$ atuavam há 5 anos ou menos na profissão (média de 7,9 anos e DP de $\pm 8,1$ anos), somente $33,1 \%$ eram servidores (Regime Jurídico Único - RJU), 58,3\% exercia suas funções nos biotérios de criação/ manutenção e $41,7 \%$ no de experimentação (88 e 63 profissionais, respectivamente); 69,5\% trabalhavam com camundongos (Mus musculus), no entanto, vários participantes trabalhavam com mais de um modelo animal, principalmente quando se tratava dos biotérios de experimentação. Além disso, cerca de $30 \%$ já sofreram algum tipo de acidente de trabalho no biotério como mordedura dos animais, queda, queimadura em autoclave e acidentes com objetos perfurocortantes, e $13,2 \%$ tiveram que ser realocados para outra área no biotério, principalmente por problemas osteomusculares.
A tabela 1 apresenta a análise sobre a associação entre a finalidade dos biotérios e os fatores de risco, segundo a percepção dos profissionais que participaram da pesquisa. Os dados mostraram que não foram detectadas associação estatisticamente significativa entre a finalidade dos biotérios e os fatores de risco biológico $(\mathrm{p}=0,827)$, químico $(\mathrm{p}=$ $0,330)$ e organizacional/psicossocial $(p=$ 0,098). Destaca-se que, em ambos os biotérios, a proporção de participantes que consideraram ser um ambiente em 'alto risco' para esses fatores foi menor.

Quanto ao fator de risco físico, foi detectada associação estatisticamente significativa entre o fator de risco físico e a finalidade dos biotérios $(\mathrm{p}<0,001)$. Entre os profissionais dos biotérios de experimentação, somente $27,0 \%$ se apresentaram em 'alto risco' físico. Em contrapartida, nos biotérios de criação, mais da metade dos participantes consideraram estar em 'alto risco' físico (55,7\%). Também foi detectada associação entre a finalidade do biotério e o fator de risco de acidente $(p=0,001)$. Ocorreu maior frequência de pessoas que se consideravam em 'alto risco' entre aqueles que trabalhavam nos biotérios de criação/manutenção (55,7\%). Do mesmo modo, a finalidade dos biotérios se mostrou associada estatisticamente com o fator de risco ergonômico. Os dados evidenciaram a maior proporção de resposta acima da mediana ('alto risco') nos biotérios de criação/manutenção (50,7 \%).

A tabela 2 apresenta a análise sobre a associação entre a finalidade dos biotérios e as implicações à saúde. O estresse e os problemas musculoesqueléticos foram os danos referidos com maior frequência pelos participantes. Em relação às análises bivariadas, não foram detectadas associações estatisticamente significativas entre a finalidade dos biotérios e as diferentes implicações à saúde provocadas e/ou agravadas pelo trabalho. No entanto, vale destacar que 'doenças de pele' e o 'estresse' apresentaram um valor de $p$ menor que 0,10 . 
Tabela 1. Consequências do desastre de janeiro de 2011 na região serrana

\begin{tabular}{|c|c|c|c|c|c|}
\hline \multirow{2}{*}{ Fatores de risco/Biotérios } & \multicolumn{2}{|c|}{ Baixo risco } & \multicolumn{2}{|c|}{ Alto risco } & \multirow{2}{*}{ Valor de $P^{\star}$} \\
\hline & N & $\%$ & $\mathrm{~N}$ & $\%$ & \\
\hline \multicolumn{6}{|l|}{ Fatores de risco Físico } \\
\hline Experimentação & 46 & 73,0 & 17 & 27,0 & \multirow{3}{*}{0,000} \\
\hline Criação/manutenção & 39 & 44,3 & 49 & 55,7 & \\
\hline Total & 85 & 56,3 & 66 & 43,7 & \\
\hline \multicolumn{6}{|l|}{ Fatores de risco biológico } \\
\hline Experimentação & 32 & 50,8 & 31 & 49,2 & \multirow{3}{*}{0,827} \\
\hline Criação/manutenção & 60 & 68,2 & 28 & 31,8 & \\
\hline Total & 92 & 60,9 & 59 & 39,1 & \\
\hline \multicolumn{6}{|l|}{ Fatores de risco químico } \\
\hline Experimentação & 33 & 52,4 & 30 & 20,6 & \multirow{3}{*}{0,330} \\
\hline Criação/manutenção & 44 & 50,6 & 43 & 18,2 & \\
\hline Total & 77 & 51,3 & 73 & 48,7 & \\
\hline \multicolumn{6}{|l|}{ Fatores de risco de acidente } \\
\hline Experimentação & 44 & 72,1 & 17 & 27,9 & \multirow{3}{*}{0,001} \\
\hline Criação/manutenção & 39 & 44,3 & 49 & 55,7 & \\
\hline Total & 83 & 55,7 & 66 & 44,3 & \\
\hline \multicolumn{6}{|l|}{ Fatores de risco ergonômico } \\
\hline Experimentação & 42 & 66,7 & 21 & 33,3 & \multirow{3}{*}{0,035} \\
\hline Criação/manutenção & 43 & 49,4 & 44 & 50,7 & \\
\hline Total & 85 & 56,7 & 66 & 43,3 & \\
\hline \multicolumn{6}{|c|}{ Fatores de risco de organizacional/psicossocial } \\
\hline Experimentação & 39 & 61,9 & 24 & 38,1 & \multirow{3}{*}{0,098} \\
\hline Criação/manutenção & 42 & 48,3 & 45 & 51,7 & \\
\hline Total & 81 & 54,0 & 69 & 46,0 & \\
\hline
\end{tabular}

Fonte: Elaboração própria.

*teste de Qui-quadrado de Pearson. 
Tabela 2. Implicações à saúde provocadas/agravadas pelo trabalho em função da finalidade dos biotérios. Saúde do trabalhador em biotérios, 2014

\begin{tabular}{|c|c|c|c|c|c|c|c|}
\hline \multirow{3}{*}{ Implicações à saúde } & \multirow{2}{*}{\multicolumn{2}{|c|}{$\begin{array}{l}\text { Frequência } \\
\text { (total) }\end{array}$}} & \multicolumn{4}{|c|}{ Finalidade dos biotérios } & \multirow{3}{*}{ Valor de P } \\
\hline & & & \multicolumn{2}{|c|}{ Criação / Manutenção } & \multicolumn{2}{|c|}{ Experimentação } & \\
\hline & $\mathbf{N}$ & $\%$ & $\mathbf{N}$ & $\%$ & $\mathbf{N}$ & $\%$ & \\
\hline Dor de cabeça & 32 & 21,2 & 13 & 15,5 & 11 & 18,6 & 0,618 * \\
\hline Problemas respiratórios & 56 & 37,1 & 25 & 30,5 & 17 & 30,9 & $0,958^{\star}$ \\
\hline Varizes & 31 & 21,9 & 14 & 16,1 & 8 & 14,5 & 0,804 * \\
\hline Problemas auditivos & 14 & 8,6 & 10 & 11,5 & 2 & 3,2 & $0,124^{\star \star}$ \\
\hline Problemas oculares & 56 & 37,1 & 18 & 23,1 & 8 & 19,5 & $0,655^{\star}$ \\
\hline Hemorroida & 8 & 5,3 & 2 & 2,3 & 4 & 6,5 & $0,234^{\star \star}$ \\
\hline Doenças renais & 9 & 6,0 & 6 & 6,8 & 1 & 1,6 & $0,241^{\star \star}$ \\
\hline Hipertensão & 24 & 15,7 & 8 & 9,8 & 6 & 10,2 & $0,935^{\star}$ \\
\hline Doenças de pele & 11 & 7,3 & 8 & 9,3 & 1 & 1,6 & $0,079^{\star \star}$ \\
\hline Dores musculares crônicas & 40 & 27,2 & 20 & 23 & 19 & 30,2 & $0,323^{*}$ \\
\hline Problemas na coluna vertebral & 58 & 37,7 & 27 & 31,8 & 23 & 39,7 & $0,331^{\star}$ \\
\hline LER ou Dort & 46 & 31,1 & 26 & 29,9 & 19 & 30,2 & $0,971^{\star}$ \\
\hline Adormecimento dos membros & 30 & 19,2 & 19 & 21,8 & 9 & 14,5 & $0,259^{\star}$ \\
\hline Depressão & 16 & 9,9 & 6 & 7 & 5 & 8,3 & $0,760^{*}$ \\
\hline Estresse & 59 & 39,1 & 36 & 42,4 & 17 & 28,8 & $0,098^{\star}$ \\
\hline Alterações de comportamento & 41 & 27,2 & 24 & 28,2 & 10 & 16,9 & $0,117^{\star}$ \\
\hline Uso frequente de medicamentos & 42 & 27,8 & 16 & 19,5 & 12 & 22,2 & $0,702^{\star}$ \\
\hline Problemas digestivos & 20 & 13,9 & 10 & 12 & 3 & 4,9 & $0,238^{\star \star}$ \\
\hline Problemas de fertilidade & 3 & 2,0 & 1 & 1,1 & 0 & 0 & 1,000 ** \\
\hline Afastamentos por saúde & 11 & 7,3 & 5 & 5,7 & 6 & 9,5 & $0,370^{*}$ \\
\hline Zoonoses & 2 & 1,3 & 0 & 0 & 1 & 1,6 & $0,420^{\star \star}$ \\
\hline
\end{tabular}

Fonte: Elaboração própria.

* teste de Qui-quadrado de Pearson.

${ }^{* *}$ teste de Fisher's.

\section{Discussão}

Quanto aos fatores de risco biológico, a ausência de associação foi atribuída aos procedimentos realizados nas áreas finalísticas dos biotérios de criação/manutenção e experimentação, local onde foi realizada a pesquisa. Os profissionais que desempenham suas atividades nessas áreas estão expostos aos agentes de risco biológicos oriundos do manejo das espécies. Além disso, estudos apontam que, além da manipulação desses agentes, os trabalhadores dos biotérios de experimentação estão sujeitos, ainda, à contaminação por materiais potencialmente infecciosos derivados das investigações científicas, o que leva a maior exposição aos riscos biológicos nesse ambiente (PRATES; RODRIGUES, 2013; CABALLERO ET AL., 2012). Em ambos os biotérios, a proporção de participantes que consideraram ser um ambiente em 'alto risco' para esses fatores de risco biológico foi baixa (tabela 1). Supõe-se que, pelo fato de os dados terem sido obtidos por meio da percepção do trabalhador, um possível 'viés de memória' ou de 
'informação' pode ter levado à subestimação das variáveis (ROUQUAYROL; SILVA, 2013).

Em relação aos fatores de risco físico, a associação e o fato dos biotérios de criação/ manutenção ter um maior percentual que consideraram estar em 'alto risco' podem ter ocorrido pelas condições das instalações dos ambientes estudados. Nesse contexto, Clough (2006) defende que instalações adequadas contribuem para a proteção aos riscos dos bioteristas, além de proporcionar uma barreira de proteção para as pessoas que se encontram fora da área do laboratório. Vale ressaltar que, no período da coleta de dados, o biotério de criação estava passando por um problema no sistema de refrigeração, o que pode ter contribuído para o resultado acima.

Da mesma forma, os fatores de acidentes se mostraram associados à finalidade do biotério, estando o biotério de criação/manutenção com maior percentual de profissionais que consideraram estar em 'alto risco'. Autores indicam que os acidentes ocorrem, sobretudo, pela falta de informação, existência de instalações e equipamentos inadequados para realizar as tarefas (CLOUGH, 2006; OLIVEIRA; DIAZ; TOLEDO, 2014), o que pode ter conduzido a esse resultado. A fim de minimizar a exposição aos riscos de acidente, bem como outros fatores de risco, os 'equipamentos de proteção individual (EPIs)' e os 'equipamentos de proteção coletiva (EPCs)' servem como elementos de apoio na segurança do pessoal e proteção do ambiente. A seleção dos tipos e o uso desses equipamentos ocorrerão em função do nível de biossegurança (NB), em que o maior NB significa a maior complexidade destes; dessa forma, faz-se necessária uma avaliação prévia dos riscos inerentes de cada atividade antes da escolha desses equipamentos (CDC, 2009). Destaca-se que EPIs são regulamentados pela Portaria $\mathrm{n}^{\circ} 25$ (NR-6), do Ministério do Trabalho, e devem estar disponíveis em número suficiente nos postos de trabalho de maneira a garantir o fornecimento para todos que necessitam utilizá-los, sendo o empregador responsável por esse fornecimento (SILVA, 2007). Os EPCs também necessitam seguir as orientações fornecidas nos manuais de referências (CDC, 2009; NRC, 2010).

A respeito dos fatores de risco ergonômico, avalia-se o fato de o biotério de criação/ manutenção ter maior percentual de profissionais que consideraram estar em 'alto risco' quando comparados ao de experimentação. Tal fato resulta da intensificação do trabalho, que advém da alta produtividade para atender às demandas, aliado à carência e afastamento por problemas de saúde dos profissionais, como também ao ambiente arquitetônico (layout) inadequado das salas destinadas ao manejo dos animais de laboratório dos biotérios de criação/manutenção.

Alguns estudos sugerem que esses fatores acarretam sobrecarga física devido à necessidade de adoção de posturas impróprias por período prolongado, bem como levantamento de cargas excessivas durante as atividades (LEBLANC ET AL., 2010; KIERMAYER ET AL., 2011). Ademais, a maioria dos biotérios estudados não contempla vários pré-requisitos recomendados pela Resolução Normativa 15, de 16 de dezembro de 2013, do Conselho Nacional de Controle de Experimentação Animal (Concea), que trata sobre a estrutura física e o ambiente de criação e utilização de roedores e lagomorfos. Como também não contempla, em muitos aspectos, a NR17, que regulamenta os aspectos ergonômicos no ambiente de trabalho, com objetivo de estabelecer parâmetros que permitam a adaptação das condições de trabalho às características psicofisiológicas dos trabalhadores, de modo a proporcionar um máximo de conforto, segurança e desempenho eficiente (BRASIL, 1990). Além de não contemplarem, em muitos aspectos, as recomendações preconizadas por normas manuais de referências disponíveis (CDC, 2009; NCR, 2010).

Quanto aos fatores de risco químico, a ausência na detecção de associação no estudo em questão corrobora os dados identificados por Majerowicz (2008B), em que os profissionais de biotérios de criação e experimentação 
estão expostos diariamente aos agentes químicos, o que oferece riscos à saúde dos trabalhadores. Entre eles, a amônia oriunda da ação das bactérias uréases presentes nos excretos dos animais são consideradas um dos principais contaminantes ambientais em biotérios. Sua concentração dentro das instalações do biotério dependerá da associação de diversos fatores como condições de temperatura, umidade relativa do ar, quantidade de trocas no ar no ambiente, taxa de ventilação, densidade de ocupação de animais na gaiola, frequência de limpeza das gaiolas de animal, volume e constituição da cama (FAITH; HESSLER, 2006). As faixas toleráveis e os níveis máximos de exposição à amônia adotados em biotérios são baseadas no valor limite de exposição para humanos recomendados pela American Conference of Governmental Industrial Hygienists (ACGIH), que é de 25 ppm para a jornada de trabalho diário de $8 \mathrm{~h}, 5$ dias por semana. No entanto, nesse ambiente, a concentração de amônia deve ser mantida tão baixa quanto possível (GREEN ET AL., 2008).

Quanto aos dos fatores de risco organizacional/psicossocial, é possível que a ausência de associação com a finalidade do biotério tenha ocorrido pela própria natureza desses fatores, que derivam da organização, gerenciamento e relações interpessoais no ambiente de trabalho (BOIX; VOGEL, 2000; FELASA, 2010). Na pesquisa, em ambos os biotérios, foi observada a prevalência de diversos fatores de risco organizacional/psicossocial, dentre os quais, destacam-se os relacionados à remuneração e vínculo institucional. A maioria dos participantes (62,3\%) tinha como forma de contratação a terceirização, muitos desses, com salários baixos, sem direito à insalubridade, o que configurava uma precarização de algumas formas de contrato de trabalho. Cabe aqui salientar que, por ser o objeto de pesquisa uma instituição pública, os profissionais dependem de concurso público para melhorar os cargos e salários. Mesmo assim, muitas medidas podem ser tomadas para promover a valorização de algumas formas de contratação (terceirização) encontradas nos biotérios.
Nas análises bivariadas, as implicações à saúde relacionadas com o trabalho não demonstraram grau de associação com a finalidade dos biotérios. Sabe-se que nesse tipo de análise não é possível distinguir que aspectos podem estar correlacionados, no entanto, acredita-se que esses resultados podem ter ocorrido porque a percepção do trabalhador está interpelada pela visão dominante na sociedade, que nega ou oculta a determinação do trabalho no processo saúde-doença (DAL ROSSO, 2008; PINA; STOTZ, 2014). Estima-se, também, que esse resultado se deve ao fato de basear-se em estudo de desenho transversal, o qual proporciona imagem pontual na relação entre as variáveis que se pretende avaliar; portanto, não se pode estabelecer uma relação temporal entre os eventos. $\mathrm{O}$ que pode ter proporcionado o 'efeito do trabalhador sadio' é um viés a ser considerado neste desenho de estudo.

Dessa forma, os profissionais da população investigada podem constituir uma seleção de bioteristas que 'sobreviveram' aos efeitos estudados, o que pode levar à subestimação das respectivas prevalências dos desfechos (MEDRONHO, 2006); ou, ainda, um viés de 'informação', por não ligar a patologia ao processo e objeto de trabalho, pode ter levado a essa subestimação (ROUQUAYROL; SILVA, 2013). Esse comportamento merece, sem dúvida, estudos de cunho qualitativo que auxiliem no seu entendimento.

Acredita-se que a presença de alguns fatores de risco foi determinante para a manifestação dessas implicações à saúde. Nesse contexto, pesquisadores acentuam que fatores de risco ergonômico podem provocar danos à saúde, comprometendo sua produtividade, segurança e saúde, sob a forma de cansaço físico, dores musculares, hipertensão arterial, alterações do sono, diabetes, doenças nervosas, taquicardia, doenças do aparelho digestivo e problemas na coluna vertebral (OLIVEIRA; BONFATTI; LIMA, 2010).

Há ainda pesquisas que indicam os agentes químicos usados nos biotérios como 
nocivos, logo, sua manipulação incorreta representa um risco ao trabalhador, produzindo alterações de ordem fisiológicas estruturais e funcionais, que contribuem para a manifestação de algumas implicações à saúde como problemas respiratórios e dores de cabeça (FAITH; HUERKAMP, 2009).

Há sinalizações que apontam a exposição às vibrações e ruídos no trabalho como fatores de risco físico, responsáveis pelos efeitos de perda da capacidade auditiva, estresse, interferência nas comunicações, aumento da tensão muscular, dores de cabeça, prejudicando as tarefas que exigem concentração mental, atenção ou velocidade e precisão dos movimentos necessários ao desempenho da função (GARCíA; GARCíA, 2004). $\mathrm{Na}$ mesma vertente, condições ambientais desfavoráveis como excesso de calor, excesso de frio, umidade relativa do ar muito alta ou baixa geram tensões no trabalho e causam desconforto, aumentando o risco de acidentes e provocando danos à saúde do trabalhador (CDC, 2009; NRC, 2010).

Já entre os fatores de risco biológico potenciais desencadeadores de doenças ocupacionais, destacam-se os microrganismos que são carreados pelos animais de laboratório, muitos naturais de sua flora, outros patogênicos aos animais e aos trabalhadores, já outros considerados oportunistas por causarem doenças em indivíduos imunodeprimidos. Ainda há, em biotérios, os animais que são propositalmente contaminados por agentes infecciosos nos experimentos, cuja manipulação incorreta pode contaminar os bioteristas (FRISSO; SOARES, 2010). Para minimização desses riscos, o Centers for Disease Controland Prevention (2009) orienta a elaboração de um manual de biossegurança específico para cada biotério, contendo informações sobre os perigos potenciais, que possuam instruções para realizar todos os procedimentos e que seja disponível, acessível e de leitura obrigatória a todos os profissionais que possuam vínculo com o biotério, incluindo os responsáveis pela manutenção das instalações e visitantes (CDC, 2009).

Assim também a falta de EPIs e EPCs, e sua utilização inadequada, considerados fatores de risco de acidente, contribui de maneira efetiva para a prevalência de algumas implicações à saúde como as 'doenças de pele', os 'problemas osteomusculares', a 'zoonose', além dos 'problemas respiratórios' já associados a outros fatores (SARGENT; GALLO, 2003; PRATES; RODRIGUES, 2013).

Além disso, as manifestações dos problemas de saúde também foram influenciadas pelos fatores de risco organizacional/ psicossocial, pois, como ratifica o Instituto Nacional de Seguridad y Higiene en el Trabajo (2008), as condições deficientes na organização de trabalho ocasionam danos ao trabalhador, como as lesões por acidente de trabalho, fadiga, insatisfação, estresse e patologias inespecíficas. Fatores psicossociais como a discriminação e assédio moral no trabalho também ocasionam danos à saúde do trabalhador (HIRIGOYEN, 2002).

\section{Conclusões}

Dada a escassez de pesquisas sobre a temática no Brasil, o estudo contribuiu, de maneira efetiva, para o processo de conhecimento da associação entre as diferentes finalidades dos biotérios e os fatores de risco ocupacionais, bem como as possíveis implicações à saúde que acometem os bioteristas, contribuindo para que esta discussão seja ampliada e se converta em propostas de ações voltadas à busca de condições dignas para essa categoria.

Pelo exposto, considerou-se urgente ação que promovam as mudanças nas operações realizadas nas instalações dos biotérios. Pode-se definir que a forma mais eficaz de minimizar a exposição aos riscos é a adoção de medidas preventivas que considere a diferença da prevalência dos fatores de risco nos biotérios de criação/ manutenção e experimentação, pois 
permite caracterizar cada situação por meio de uma avaliação minuciosa do ambiente e das condições de trabalho. Nesse contexto, o envolvimento das partes interessadas, ou seja, gestores, empregadores e trabalhadores, faz-se primordial para subsidiar a elaboração de estratégias, buscando-se a melhoria efetiva das condições de saúde e de trabalho, bem como promover ações direcionadas à intervenção dos problemas levantados aqui a partir de um planejamento político eficiente.

\section{Referências}

BOIX, P.; VOGEL. La evaluación de riesgos en los lugares de trabajo: guía para una intervención sindical. Bruxelas: BTS, 2000.

BRASIL. Ministério da Ciência, Tecnologia e Inovação. Resolução Normativa ${ }^{\circ} 15$, de 16 de dezembro de 2013. Diário Oficial [da] União, Brasília, DF, 17 dez. 2013. Disponível em: <http://www.lex.com.br/legis_25182844_RESOLUCAO_NORMATIVA_N_15_ DE_16_DE_DEZEMBRO_DE_2013.aspx>. Acesso em: 18 dez. 2014.

Ministério do Trabalho e Emprego. Portaria ${ }^{\circ}$ 3751 de 23 de novembro de 1990. Diário Oficial [da] União, Brasília, DF, 26 de nov. de 1990. Disponível em: <http:// www.fclar.unesp.br/Home/Instituicao/Administracao/ CIPA/nr_17.pdf $>$. Acesso em: 4 mar. 2015.

CABALLERO, M. L. et al. Characterization of occupational sensitization by multiallergen immunoblotting in workers exposed to laboratory animals. Annals of Allergy, Asthma \&t Immunology, Philadelphia, v. 108, n. 3, p. 178-181, 2012.

CARDOSO, T. A.; SILVA, I. Biossegurança no Manejo de Animais. In: CARDOSO, T. A. O.; NAVARRO, M. B. M. A. A ciência entre bichos e grilos: reflexões e ações da biossegurança na pesquisa com animais. São Paulo: Hucitec, Rio de Janeiro: Faperj, 2007, p. 229-257.

\section{CENTERS FOR DISEASE CONTROL AND}

PREVENTION (CDC). Biosafety in microbiological and biomedical laboratories. 4. ed. Atlanta: U.S. Department of Health and Human Services, 2009.

CLOUGH, G. The animal house: design, equipment and environmental control. In: POOLE, T. The UFAW handbook on the care and management of laboratory animals. 7. ed. [S. 1.]: Blackwell Science, 2006. p. 97-136.

COSTA, F. G. Emergências em Biotérios. In: MOLINARO, E. M.; MAJEROWICZ, J. VALLE, S. (Org.). Biossegurança em Biotérios. Rio de Janeiro: Interciência, 2008, p. 193-205.

DAL ROSSO, S. Mais trabalho: a intensificação do labor na sociedade contemporânea. São Paulo: Bomtempo, 2008.

FAITH, R. E.; HESSLER, J. R. Housing and environment. In: SUCKOW, M. A. et al. (Ed.). The laboratory rat. 2. ed. London: Elsevier, 2006. p. 303-337.

FAITH, R. E.; HUERKAMP, M. J. Environmental considerations for research animals. In: HESSLER, J. R.; LEHNER, N. D. M. (Ed.). Planning and Designing Research Animal Facilities. 1. ed. Londres: Academic Press, 2009, p. 59-84. 
FEDERATION OF EUROPEAN LABORATORY ANIMAL SCIENCE ASSOCIATIONS (FELASA). Guidelines for Continuing Education for Persons Involved in Animal Experiments: Recommendations of a FELASA Working Group. United Kingdom, 2010. Disponível em: <http://www.felasa.eu/media/uploads/ Guidelines\%20for\%20Continuing\%20Education $\% 20$ of\%20Animal\%20Technologists_\%20final.pdf >. Acesso em: 21 jan. 2015.

FRISSO, M. A. A.; SOARES, B. E. C. Ensino em Biossegurança: educação e sensibilização do profissional de biotério para as práticas seguras de experimentação animal. Revista Eletrônica Ensino, Saúde e Ambiente, São Paulo, v. 3, n. 3, p. 80-95, dez. 2010.

GARCÍA GARCÍA, A. M. Agentes físicos. In: GARCÍA BENAVIDES, F.; RUIZFRUTOS, C.; GARCÍA GARCÍA, A. M. Salud Laboral: conceptos y técnicas para la prevención de riesgos laborales. 2. ed. Barcelona: Masson, 2004.

GREEN, A. R. et al. Development and application of a novel environmental preference chamber for assessing responses of laboratory mice to atmospheric ammonia. The Journal of the American Association for Laboratory Animal Science, v. 47, p. 49-56, mar. 2008.

HIRIGOYEN, M. F. Mal-estar no trabalho: redefinindo o assédio moral. Rio de Janeiro: Bertrand Brasil, 2002.

\section{INSTITITO NACIONAL DE SEGURIDADE} E HIGIENE EM EL TRABAJO. Manual de Procedimientos de Prevención de Riesgos Laborales: guía de elaboración. Espanha, 2002. Disponível em: $<$ https://www.cnae.com/ficheros/files/prl/Manual_ procedimientos.pdf $>$. Acesso em: 20 nov. 2013.

KIERMAYER, C. et al. Musculoskeletal Load in and Highly Repetitive Actions of Animal Facility Washroom Employees. Journal of the American Association for Laboratory Animal Science, v. 50, n. 5, p. 665-674, set. 2011.

LEBLANC, S. et al. Application of Ergonomics to animal facility operations. ALN Magazine, v. 9, n. 7, 2010. Disponível em: <https://www.alnmag.com/ article/2010/10/application-ergonomics-animal-facility-operations>. Acesso em: 20 nov. 2013.

MAJEROWICZ, J. Considerações Básicas em Bioterismo. In: MOLINARO, E. M.; MAJEROWICZ, J.; VALLE, S. (Org.). Biossegurança em Biotérios. Rio de Janeiro: Interciência, 2008a, p.1-18.

MAJEROWICZ, J. Riscos químicos. In: MOLINARO, E. M.; MAJEROWICZ, J.; VALLE, S. (Org.). Biossegurança em Biotérios. Rio de Janeiro: Interciência, 2008b, p. 101-117.

MEDRONHO, R. A. Epidemiologia. São Paulo: Atheneu, 2006.

MEZADRI, T. J.; TOMAZ, V. A.; AMARAL, V. L. L. Animais de Laboratório: cuidados na iniciação experimental. Florianópolis: UFSC, 2004.

NATIONAL RESEARCH COUNCIL (NRC). Guide for the Care and Use of Laboratory Animals: Committee for the Update of the Guide for the Care and Use of Laboratory Animals. 8. ed. Washington: National Academy, 2010.

NOUROUDINE, A. Risco e atividades humanas: acerca da possível positividade aí presente. In: FIGUEIREDO, M. et al. (Org.). Labirintos do trabalho: interrogações e olhares sobre o trabalho vivo. Rio de Janeiro: DP\&A, 2004. p. 37-62.

OLIVEIRA, A. C.; DIAZ, M. E. P.; TOLEDO, A. D. Acidentes de trabalho com materiais perfurocortantes entre a equipe multiprofissional de uma unidade de emergência. Revista Temas em Saúde, João Pessoa, v. 14, n. 1, p. 205-217, jul./set. 2014.

OLIVEIRA, S. S.; BONFATTI, R. J.; LIMA, M. B. Ergonomia em Laboratórios. In: TEIXEIRA, P.; VALLE, S. (Org:). Biossegurança: uma abordagem multidisciplinar. 2. ed. Rio de Janeiro: Fiocruz, 2010. p. 239-257.

PINA, J. A.; STOTZ, E. N. Intensificação do trabalho e saúde do trabalhador: uma abordagem teórica. Revista Brasileira de Saúde Ocupacional, São Paulo, v. 39, n. 130, p. 150-160, 2014. 
PORTO, M. F. S. Análise de riscos nos locais de trabalho: conhecer para transformar. Cadernos de Saúde do Trabalhador, Rio de Janeiro, v. 3, jun. 2000.

PRATES, F. M.; RODRIGUES L. D. Seção de Experimentação. In: NEVES, S. M. P. et al. São Paulo: FCF-IQ/USP, 2013, p. 101-111.

ROUQUAYROL, M. Z.; SILVA, M. C. Epidemiologia \&t Saúde. 7. ed. Rio de Janeiro: Medbook, 2013.

SARGENT E. V.; GALLO, F. Use of personal protective equipment for respiratory protection. Ilar Journal, v. 44, n. 1, p. 52-56, 2003.
SILVA, F. H. A. L. Equipamentos de Proteção com Animais de Laboratório. In: CARDOSO, T. A. O; NAVARRO, M. B. M. A. A Ciência entre Bichos e Grilos: reflexões e ações da biossegurança na pesquisa com animais. São Paulo: Hucitec; Rio de Janeiro: Faperj, 2007, p. 186-191.

Recebido para publicação em agosto de 2016

Versão final em janeiro de 2017

Conflito de interesses: inexistente

Suporte financeiro: não houve 